\title{
GLOBAL EXISTENCE OF PERIODIC SOLUTIONS IN \\ A SIMPLIFIED FOUR-NEURON BAM NEURAL NETWORK MODEL WITH MULTIPLE DELAYS
}

XIANG-PING YAN AND WAN-TONG LI

Received 8 September 2005; Accepted 5 December 2005

We consider a simplified bidirectional associated memory (BAM) neural network model with four neurons and multiple time delays. The global existence of periodic solutions bifurcating from Hopf bifurcations is investigated by applying the global Hopf bifurcation theorem due to $\mathrm{Wu}$ and Bendixson's criterion for high-dimensional ordinary differential equations due to Li and Muldowney. It is shown that the local Hopf bifurcation implies the global Hopf bifurcation after the second critical value of the sum of two delays. $\mathrm{Nu}-$ merical simulations supporting the theoretical analysis are also included.

Copyright (c) 2006 X.-P. Yan and W.-T. Li. This is an open access article distributed under the Creative Commons Attribution License, which permits unrestricted use, distribution, and reproduction in any medium, provided the original work is properly cited.

\section{Introduction}

In this paper, we consider the global existence of periodic solutions bifurcating from the Hopf bifurcations to the simplified bidirectional associated memory (BAM) neural network model with four neurons and two differential transmission delays:

$$
\begin{gathered}
\dot{u}_{1}(t)=-\mu_{1} u_{1}(t)+c_{21} f_{1}\left(u_{2}\left(t-\tau_{2}\right)\right)+c_{31} f_{1}\left(u_{3}\left(t-\tau_{2}\right)\right)+c_{41} f_{1}\left(u_{4}\left(t-\tau_{2}\right)\right), \\
\dot{u}_{2}(t)=-\mu_{2} u_{2}(t)+c_{12} f_{2}\left(u_{1}\left(t-\tau_{1}\right)\right), \\
\dot{u}_{3}(t)=-\mu_{3} u_{3}(t)+c_{13} f_{3}\left(u_{1}\left(t-\tau_{1}\right)\right), \\
\dot{u}_{4}(t)=-\mu_{4} u_{4}(t)+c_{14} f_{4}\left(u_{1}\left(t-\tau_{1}\right)\right),
\end{gathered}
$$

which implies that there is only one neuron on the $I$-layer whose state and decay rate are, respectively, $u_{1}(t)$ and $\mu_{1}$ and three neurons on the $J$-layer whose states and decay rates are, respectively, $u_{k}(t)$ and $\mu_{k}(k=2,3,4)$, the time delay from the $I$-layer to another $J$ layer is $\tau_{1}$, while the delay from the $J$-layer back to the $I$-layer is $\tau_{2}$, the connected weights 


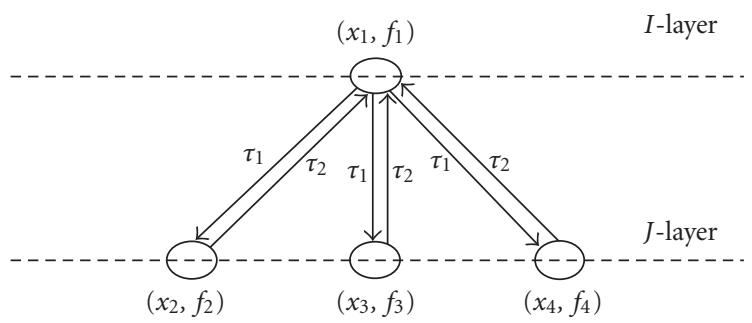

Figure 1.1. Graph of the architecture of the model (1.1).

and the activation function from the neuron $u_{k}$ to $u_{1}$ are, respectively, $c_{k 1}$ and $f_{1}$, and from $u_{1}$ to $u_{k}$ are $c_{1 k}$ and $f_{k}(k=2,3,4)$, respectively (see Figure 1.1 ).

It is well known that periodic solutions can arise through the Hopf bifurcation in delay differential equations. For the existence of local periodic solutions bifurcating form Hopf bifurcations in delayed neural networks, we refer to $[1,2,4,3,9,10]$. Recently, Yan and $\mathrm{Li}$ [12] considered the system (1.1) and obtained sufficient conditions ensuring the absolute stability, conditional stability of the zero equilibrium, and the existence of Hopf bifurcations by using the results due to Ruan and Wei [7] and some analysis techniques. Furthermore, by applying the normal form theory and the center manifold theorem due to Hassard et al. [5], we also obtained the formulae determining the direction and stability of the Hopf bifurcation.

In general, the periodic solutions bifurcating from Hopf bifurcations are local. It is well known that the study on dynamical systems not only involve a local dynamics, but also involve a global one. In particular, the global existence of periodic solutions, which can arise through the local Hopf bifurcation in delayed systems, is of great interest. Recently, Wei and Li [8] established the global existence of a tri-neuron ring model with delays. But up to now, to the best of our knowledge, there are no results on the global existence of periodic solutions of the neural networks with four or more neurons and delays.

Based on the results obtained by Yan and Li [12] regarding the existence of periodic solutions bifurcating from local Hopf bifurcations of (1.1), the purpose of this paper is to investigate the global existence of multiple periodic solutions for (1.1) by using a global Hopf bifurcation result on functional differential equations due to $\mathrm{Wu}$ [11] and the Bendixsons criterion for higher-dimensional ordinary differential equations due to $\mathrm{Li}$ and Muldowney [6].

This paper is organized as follows. In Section 2, we introduce the local Hopf bifurcation results of the model (1.1) obtained by Yan and $\mathrm{Li}$ [12]. In Section 3, we show that the local Hopf bifurcation of system (1.1) implies the global Hopf bifurcation after the second critical value of the sum of two delays by using a global Hopf bifurcation theorem due to $\mathrm{Wu}[11]$ and a Bendixson's criterion for high-dimensional ordinary differential equations due to Li and Muldowney [6]. In Section 4, the model (1.1) with special activation functions $f_{k}(u)=\tanh (u)(k=1,2,3,4)$ is studied and some numerical simulations are presented. 


\section{Results on local Hopf bifurcations}

In this section, we will list some results in [12] to establish the global existence of the nonconstant periodic solutions.

Throughout this section, we make the following hypothesis on the activation functions $f_{k}(k=1,2,3,4)$.

$\left(\mathrm{H}_{1}\right) f_{k} \in C^{1}(\mathbb{R}, \mathbb{R}), f_{k}(0)=0$, and the origin $(0,0,0,0)$ is the unique equilibrium of system (1.1).

Under the assumption $\left(\mathrm{H}_{1}\right)$, let $u_{k}(t)(k=1,2,3,4)$ be defined, respectively, by $u_{1}(t)=$ $x_{1}\left(t-\tau_{1}\right), u_{k}(t)=x_{k}(t)(k=2,3,4)$, and $\tau=\tau_{1}+\tau_{2}$. Then system (1.1) reduces to

$$
\begin{aligned}
\dot{u}_{1}(t)=-\mu_{1} u_{1}(t)+ & c_{21} f_{1}\left(u_{2}(t-\tau)\right)+c_{31} f_{1}\left(u_{3}(t-\tau)\right)+c_{41} f_{1}\left(u_{4}(t-\tau)\right), \\
\dot{u}_{2}(t) & =-\mu_{2} u_{2}(t)+c_{12} f_{2}\left(u_{1}(t)\right), \\
\dot{u}_{3}(t) & =-\mu_{3} u_{3}(t)+c_{13} f_{3}\left(u_{1}(t)\right), \\
\dot{u}_{4}(t) & =-\mu_{4} u_{4}(t)+c_{14} f_{4}\left(u_{1}(t)\right) .
\end{aligned}
$$

The linearized system of $(2.1)$ at the equilibrium $(0,0,0,0)$ is

$$
\begin{gathered}
\dot{u}_{1}(t)=-\mu_{1} u_{1}(t)+\alpha_{21} u_{2}(t-\tau)+\alpha_{31} u_{3}(t-\tau)+\alpha_{41} u_{4}(t-\tau), \\
\dot{u}_{2}(t)=-\mu_{2} u_{2}(t)+\alpha_{12} u_{1}(t), \\
\dot{u}_{3}(t)=-\mu_{3} u_{3}(t)+\alpha_{13} u_{1}(t), \\
\dot{u}_{4}(t)=-\mu_{4} u_{4}(t)+\alpha_{14} u_{1}(t),
\end{gathered}
$$

where $\alpha_{k 1}=c_{k 1} f_{1}^{\prime}(0)(k=2,3,4)$ and $\alpha_{1 l}=c_{1 l} f_{l}^{\prime}(0)(l=2,3,4)$, and the corresponding characteristic equation of system (2.2) is

$$
\operatorname{det}\left[\begin{array}{cccc}
\lambda+\mu_{1} & -\alpha_{21} e^{-\lambda \tau} & -\alpha_{31} e^{-\lambda \tau} & -\alpha_{41} e^{-\lambda \tau} \\
-\alpha_{12} & \lambda+\mu_{2} & 0 & 0 \\
-\alpha_{13} & 0 & \lambda+\mu_{3} & 0 \\
-\alpha_{14} & 0 & 0 & \lambda+\mu_{4}
\end{array}\right]=0 \text {, }
$$

that is,

$$
\begin{aligned}
& \lambda^{4}+\left(\mu_{1}+\mu_{2}+\mu_{3}+\mu_{4}\right) \lambda^{3}+\left(\mu_{1} \mu_{2}+\mu_{1} \mu_{3}+\mu_{1} \mu_{4}+\mu_{2} \mu_{3}+\mu_{2} \mu_{4}+\mu_{3} \mu_{4}\right) \lambda^{2} \\
& +\left(\mu_{1} \mu_{2} \mu_{3}+\mu_{1} \mu_{2} \mu_{4}+\mu_{1} \mu_{3} \mu_{4}+\mu_{2} \mu_{3} \mu_{4}\right) \lambda+\mu_{1} \mu_{2} \mu_{3} \mu_{4} \\
& -\left\{\left(\alpha_{12} \alpha_{21}+\alpha_{13} \alpha_{31}+\alpha_{14} \alpha_{41}\right) \lambda^{2}\right. \\
& +\left[\alpha_{12} \alpha_{21}\left(\mu_{3}+\mu_{4}\right)+\alpha_{13} \alpha_{31}\left(\mu_{2}+\mu_{4}\right)+\alpha_{14} \alpha_{41}\left(\mu_{2}+\mu_{3}\right)\right] \lambda \\
& \left.+\alpha_{12} \alpha_{21} \mu_{3} \mu_{4}+\alpha_{13} \alpha_{31} \mu_{2} \mu_{4}+\alpha_{14} \alpha_{41} \mu_{2} \mu_{3}\right\} e^{-\lambda \tau}=0 .
\end{aligned}
$$


4 A BAM neural network with delays

Set

$$
\begin{gathered}
a_{3}=\mu_{1}+\mu_{2}+\mu_{3}+\mu_{4}>0 \\
a_{2}=\mu_{1} \mu_{2}+\mu_{1} \mu_{3}+\mu_{1} \mu_{4}+\mu_{2} \mu_{3}+\mu_{2} \mu_{4}+\mu_{3} \mu_{4}>0 \\
a_{1}=\mu_{1} \mu_{2} \mu_{3}+\mu_{1} \mu_{2} \mu_{4}+\mu_{1} \mu_{3} \mu_{4}+\mu_{2} \mu_{3} \mu_{4}>0 \\
a_{0}=\mu_{1} \mu_{2} \mu_{3} \mu_{4}>0 \\
b_{2}=-\left(\alpha_{12} \alpha_{21}+\alpha_{13} \alpha_{31}+\alpha_{14} \alpha_{41}\right), \\
b_{1}=-\left[\alpha_{12} \alpha_{21}\left(\mu_{3}+\mu_{4}\right)+\alpha_{13} \alpha_{31}\left(\mu_{2}+\mu_{4}\right)+\alpha_{14} \alpha_{41}\left(\mu_{2}+\mu_{3}\right)\right] \\
b_{0}=-\left(\alpha_{12} \alpha_{21} \mu_{3} \mu_{4}+\alpha_{13} \alpha_{31} \mu_{2} \mu_{4}+\alpha_{14} \alpha_{41} \mu_{2} \mu_{3}\right)
\end{gathered}
$$

and

$$
\begin{aligned}
a= & a_{3}^{2}-2 a_{2}=\mu_{1}^{2}+\mu_{2}^{2}+\mu_{3}^{2}+\mu_{4}^{2}>0, \\
b= & 2 a_{0}+a_{2}^{2}-2 a_{1} a_{3}-b_{2}^{2} \\
= & \mu_{1}^{2} \mu_{2}^{2}+\mu_{1}^{2} \mu_{3}^{2}+\mu_{1}^{2} \mu_{4}^{2}+\mu_{2}^{2} \mu_{3}^{2}+\mu_{2}^{2} \mu_{4}^{2}+\mu_{3}^{2} \mu_{4}^{2}-\left(\alpha_{12} \alpha_{21}+\alpha_{13} \alpha_{31}+\alpha_{14} \alpha_{41}\right)^{2}, \\
c= & a_{1}^{2}-2 a_{0} a_{2}+2 b_{0} b_{2}-b_{1}^{2}=\mu_{1}^{2} \mu_{2}^{2} \mu_{3}^{2}+\mu_{1}^{2} \mu_{2}^{2} \mu_{4}^{2}+\mu_{1}^{2} \mu_{3}^{2} \mu_{4}^{2}+\mu_{2}^{2} \mu_{3}^{2} \mu_{4}^{2} \\
& +2\left(\alpha_{12} \alpha_{21}+\alpha_{13} \alpha_{31}+\alpha_{14} \alpha_{41}\right)\left(\alpha_{12} \alpha_{21} \mu_{3} \mu_{4}+\alpha_{13} \alpha_{31} \mu_{2} \mu_{4}+\alpha_{14} \alpha_{41} \mu_{2} \mu_{3}\right) \\
& -\left[\alpha_{12} \alpha_{21}\left(\mu_{3}+\mu_{4}\right)+\alpha_{13} \alpha_{31}\left(\mu_{2}+\mu_{4}\right)+\alpha_{14} \alpha_{41}\left(\mu_{2}+\mu_{3}\right)\right]^{2}, \\
d= & a_{0}^{2}-b_{0}^{2}=\mu_{1}^{2} \mu_{2}^{2} \mu_{3}^{2} \mu_{4}^{2}-\left(\alpha_{12} \alpha_{21} \mu_{3} \mu_{4}+\alpha_{13} \alpha_{31} \mu_{2} \mu_{4}+\alpha_{14} \alpha_{41} \mu_{2} \mu_{3}\right)^{2},
\end{aligned}
$$

and assume that $a_{k}, b_{l}(k=0,1,2,3 ; l=0,1,2)$ satisfy the condition $\left(\mathrm{H}_{2}\right)$.

$\left(\mathrm{H}_{2}\right) a_{0}+b_{0}>0, a_{1}+b_{1}>0, a_{3}>0$, and

$$
a_{3}\left[\left(a_{1}+b_{1}\right)\left(a_{2}+b_{2}\right)-a_{3}\left(a_{0}+b_{0}\right)\right]>\left(a_{1}+b_{1}\right)^{2} .
$$

Let

$$
h(z)=z^{4}+a z^{3}+b z^{2}+c z+d,
$$

and define

$$
p=\frac{8 b-3 a^{2}}{16}, \quad q=\frac{a^{3}-4 a b+8 c}{32}, \quad D_{0}=\frac{q^{2}}{4}+\frac{p^{3}}{27} \text {. }
$$


Then, from the sign of $D_{0}$, we have

$$
\begin{gathered}
z_{1}^{*}=-\frac{a}{4}+\sqrt[3]{-\frac{q}{2}+\sqrt{D_{0}}}+\sqrt[3]{-\frac{q}{2}-\sqrt{D_{0}}} \quad \text { if } D_{0}>0, \\
z_{2}^{*}=\max \left\{-\frac{a}{4}-2 \sqrt[3]{\frac{q}{2}},-\frac{a}{4}+\sqrt[3]{\frac{q}{2}}\right\} \quad \text { if } D_{0}=0, \\
z_{3}^{*}=\max \left\{r_{1}^{*}, r_{2}^{*}, r_{3}^{*}\right\} \quad \text { if } D_{0}<0,
\end{gathered}
$$

where

$$
r_{1}^{*}=-\frac{a}{4}+2 \operatorname{Re}\{\alpha\}, \quad r_{2}^{*}=-\frac{a}{4}+2 \operatorname{Re}\{\alpha \varepsilon\}, \quad r_{3}^{*}=-\frac{a}{4}+2 \operatorname{Re}\{\alpha \bar{\varepsilon}\},
$$

and $\alpha$ is one of the cubic roots of the complex number $-q / 2+\sqrt{D_{0}}$ and $\varepsilon=-1 / 2+$ $(\sqrt{3} / 2) i$.

In the following, we assume that the following condition holds:

$\left(\mathrm{H}_{3}\right)$ (i) $z_{1}^{*}>0$ and $h\left(z_{1}^{*}\right)<0$ when $D_{0}>0$;

(ii) $z_{2}^{*}>0$ and $h\left(z_{2}^{*}\right)<0$ when $D_{0}=0$;

(iii) $z_{3}^{*}>0$ and $h\left(z_{3}^{*}\right)<0$ when $D_{0}<0$.

Under the hypothesis $\left(\mathrm{H}_{3}\right)$, the equation $h(z)=0$ has at least one positive root $z_{0}$, and denote $\omega_{0}=\sqrt{z_{0}}$. Define

$$
\tau_{j}=\frac{1}{\omega_{0}}\left[\arccos \left(\frac{\Delta_{1}}{\Delta}\right)+2 j \pi\right], \quad j=0,1,2, \ldots
$$

where

$$
\begin{gathered}
\Delta_{1}=b_{2} \omega_{0}^{6}+\left(a_{3} b_{1}-a_{2} b_{2}-b_{0}\right) \omega_{0}^{4}+\left(a_{0} b_{2}+a_{2} b_{0}-a_{1} b_{1}\right) \omega_{0}^{2}-a_{0} b_{0} \\
\Delta=b_{2}^{2} \omega_{0}^{4}+\left(b_{1}^{2}-2 b_{0} b_{2}\right) \omega_{0}^{2}+b_{0}^{2} .
\end{gathered}
$$

From [12, Theorem 3.1], we have the following result.

Lemma 2.1. Let $a_{k}, b_{l}(k=0,1,2,3 ; l=0,1,2) ; a, b, c, d$, and $\tau_{j}$ be defined, respectively, $b y$ (2.5), (2.6), and (2.12). Suppose that $\left(\mathrm{H}_{1}\right)$ and $\left(\mathrm{H}_{2}\right)$ are satisfied, and that $d<0$ or $d \geq 0$ and one of the conditions in $\left(\mathrm{H}_{3}\right)$ holds. In addition, the positive root $z_{0}$ of (2.5) satisfies $h^{\prime}\left(z_{0}\right) \neq 0$. Then the equilibrium $(0,0,0,0)$ of $(2.1)$ is asymptotically stable when $\tau \in\left[0, \tau_{0}\right)$, and unstable when $\tau>\tau_{0}$. Moreover, at $\tau=\tau_{j}, j=0,1,2, \ldots, \pm i \omega_{0}$ is a pair of simple imaginary roots of (2.5), and (2.1) undergoes Hopf bifurcation near $(0,0,0,0)$.

\section{Global existence of periodic solutions}

In this section, we will investigate the global existence of periodic solutions bifurcating from the equilibrium $(0,0,0,0)$ of system $(2.1)$ when $\tau=\tau_{j}(j=0,1,2, \ldots)$ by applying a global Hopf bifurcation result due to Wu [11] and a Bendixson's criterion for highdimensional ordinary differential equations due to Li and Muldowney [6].

Throughout this section, we need to impose the following condition on $f_{k}$. 
$\left(\mathrm{H}_{4}\right) f_{k} \in C^{2}(\mathbb{R}, \mathbb{R})$ and there exists a constant $L>0$ such that

$$
\left|f_{k}(u)\right| \leq L \quad \forall u \in \mathbb{R}, k=1,2,3,4
$$

Let $X=C\left([-\tau, 0], \mathbb{R}^{4}\right)$ and let $u_{t}=\left(u_{t}^{(1)}, u_{t}^{(2)}, u_{t}^{(3)}, u_{t}^{(4)}\right)^{\mathrm{T}} \in X$ be defined as $u_{t}(s)=u(t+$ $s)$ for $t \geq 0$ and $s \in[-\tau, 0]$. Then system (2.1) can be rewritten as the following functional differential equation:

$$
\dot{u}(t)=F\left(u_{t}, \tau, p\right)
$$

parameterized by two real parameters $(\tau, p) \in \mathbb{R}_{+} \times \mathbb{R}_{+}$, where $\mathbb{R}_{+}=[0, \infty)$. It is obvious that (3.2) has a unique equilibrium $(0,0,0,0)$ under the assumption $\left(\mathrm{H}_{1}\right)$ and easy to see that the mapping $F: X \times \mathbb{R}_{+} \times \mathbb{R}_{+}$is completely continuous under the condition $\left(\mathrm{H}_{4}\right)$. If we restrict $F$ to the subspace of constant functions $u$, then $F$ is identified with $\mathbb{R}^{4}$ and thus

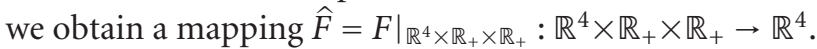

Let $\bar{u}_{0} \in X$ be the constant mapping with value $u_{0} \in \mathbb{R}^{4}$. The point $\left(\bar{u}_{0}, \tau_{0}, p_{0}\right)$ is called a stationary solution of $(3.2)$ if $F\left(\bar{u}_{0}, \tau_{0}, p_{0}\right)=0$. From the assumption $\left(\mathrm{H}_{4}\right)$ we know easily that the following condition regarding the mapping $\widehat{F}$ holds.

(A1) $\hat{F} \in C^{2}\left(\mathbb{R}^{4} \times \mathbb{R}_{+} \times \mathbb{R}_{+}, \mathbb{R}^{4}\right)$.

It follows from system $(2.1)$ and $\left(\mathrm{H}_{2}\right)$ that

$$
\begin{aligned}
\operatorname{det} D_{u} \hat{F}(0, \tau, p) & =\operatorname{det}\left[\begin{array}{cccc}
-\mu_{1} & \alpha_{21} & \alpha_{31} & \alpha_{41} \\
\alpha_{12} & -\mu_{2} & 0 & 0 \\
\alpha_{13} & 0 & -\mu_{3} & 0 \\
\alpha_{14} & 0 & 0 & -\mu_{4}
\end{array}\right] \\
& =\mu_{1} \mu_{2} \mu_{3} \mu_{4}-\left(\alpha_{12} \alpha_{21} \mu_{3} \mu_{4}+\alpha_{13} \alpha_{31} \mu_{2} \mu_{4}+\alpha_{14} \alpha_{41} \mu_{2} \mu_{3}\right)=a_{0}+b_{0}>0 .
\end{aligned}
$$

Therefore, we have the following property on the linear operator $D_{u} \widehat{F}(u, \tau, p)$.

(A2) $D_{u} \hat{F}(u, \tau, p)$ is an isomorphism at the zero equilibrium, $(\tau, p) \in \mathbb{R}_{+} \times \mathbb{R}_{+}$.

From (A1), (A2), and the implicit function theorem, for the stationary solution $\left(0, \tau_{j}\right.$, $\left.2 \pi / \omega_{0}\right), j=0,1,2, \ldots$, there exist $\varepsilon_{0}>0$ and a $C^{1}$ mapping $y: B_{\varepsilon_{0}}\left(\tau_{j}, 2 \pi / \omega_{0}\right) \rightarrow \mathbb{R}^{4}$ such that $\hat{F}(y(\tau, p), \tau, p)=0$ for $(\tau, p) \in B_{\varepsilon_{0}}\left(\tau_{j}, 2 \pi / \omega_{0}\right)=\left(\tau_{j}-\varepsilon_{0}, \tau_{j}+\varepsilon_{0}\right) \times\left(2 \pi / \omega_{0}-\varepsilon_{0}, 2 \pi / \omega_{0}\right.$ $\left.+\varepsilon_{0}\right)$.

Furthermore, it is easy to observe that the following result is true.

(A3) $F(\varphi, \tau, p)$ is differential with respect to $\varphi$.

The characteristic matrix of (3.2) at a stationary solution $\left(\bar{u}, \tau_{0}, p_{0}\right)$, where $\bar{u}=\left(\bar{u}^{(1)}\right.$, $\left.\bar{u}^{(2)}, \bar{u}^{(3)}, \bar{u}^{(4)}\right)^{\mathrm{T}} \in \mathbb{R}^{4}$, is taken as the following form:

$$
\triangle(\bar{u}, \tau, p)(\lambda)=\lambda I-D_{\varphi} F\left(\bar{u}, \tau_{0}, p_{0}\right)\left(e^{\lambda \cdot I} I\right)
$$


that is,

$$
\begin{aligned}
& \Delta(\bar{u}, \tau, p)(\lambda) \\
& =\left[\begin{array}{cccc}
\lambda+\mu_{1} & -c_{21} f_{1}^{\prime}\left(\bar{u}^{(2)}\right) e^{-\lambda \tau} & -c_{31} f_{1}^{\prime}\left(\bar{u}^{(3)}\right) e^{-\lambda \tau} & -c_{41} f_{1}^{\prime}\left(\bar{u}^{(4)}\right) e^{-\lambda \tau} \\
-c_{12} f_{2}^{\prime}\left(\bar{u}^{(1)}\right) & \lambda+\mu_{2} & 0 & 0 \\
-c_{13} f_{3}^{\prime}\left(\bar{u}^{(1)}\right) & 0 & \lambda+\mu_{3} & 0 \\
-c_{14} f_{4}^{\prime}\left(\bar{u}^{(1)}\right) & 0 & 0 & \lambda+\mu_{4}
\end{array}\right],
\end{aligned}
$$

and the zeros of det $\triangle(\bar{u}, \tau, p)(\lambda)=0$ are called the characteristic roots. Note that (A2) implies that $\lambda=0$ is not a characteristic root of equilibrium of (3.2). Clearly, the characteristic matrix $\triangle(y(\tau, p), \tau, p)(\lambda)$ is continuous in $(\tau, p, \lambda) \in B_{\varepsilon_{0}}\left(\tau_{j}, 2 p / \omega_{0}\right) \times \mathbb{C}$.

A stationary solution $\left(\bar{u}_{0}, \tau_{0}, p_{0}\right)$ of $(3.2)$ is called a center if $\operatorname{det} \triangle\left(\bar{u}, \tau_{0}, p_{0}\right)\left(i\left(2 m \pi / p_{0}\right)\right)$ $=0$ for some positive integer $m$ or the equation $\operatorname{det} \triangle\left(\bar{u}, \tau_{0}, p_{0}\right)(\lambda)=0$ has purely imaginary characteristic roots of the form $i\left(2 m \pi / p_{0}\right)$ for some positive integer $m$. A center $\left(\bar{u}_{0}, \tau_{0}, p_{0}\right)$ is said to be isolated if it is the only center in some neighborhood of $\left(\bar{u}_{0}, \tau_{0}, p_{0}\right)$. From (3.5), we can see

$$
\operatorname{det} \Delta(0, \tau, p)(\lambda)=\left[\begin{array}{cccc}
\lambda+\mu_{1} & -\alpha_{21} e^{-\lambda \tau} & -\alpha_{31} e^{-\lambda \tau} & -\alpha_{41} e^{-\lambda \tau} \\
-\alpha_{12} & \lambda+\mu_{2} & 0 & 0 \\
-\alpha_{13} & 0 & \lambda+\mu_{3} & 0 \\
-\alpha_{14} & 0 & 0 & \lambda+\mu_{4}
\end{array}\right]=0 .
$$

Note that (3.6) is the same as (2.3) and (2.4), therefore, it is easily to see that

$$
\left(0, \tau_{j}, \frac{2 \pi}{\omega_{0}}\right), \quad j=0,1,2, \ldots
$$

is an isolated center, and there exist $\varepsilon \in\left(0, \varepsilon_{0}\right), \delta \in\left(0, \varepsilon_{0}\right)$, and a smooth curve $\lambda:\left(\tau_{j}-\right.$ $\left.\delta, \tau_{j}+\delta\right) \rightarrow \mathbb{C}$ such that $\operatorname{det} \triangle\left(0, \tau, 2 \pi / \omega_{0}\right)(\lambda(\tau))=0,\left|\lambda(\tau)-i \omega_{0}\right|<\varepsilon$, for all $\tau \in\left[\tau_{j}-\right.$ $\left.\delta, \tau_{j}+\delta\right]$ and

$$
\lambda\left(\tau_{j}\right)=i \omega_{0},\left.\quad \operatorname{Re} \frac{d \lambda}{d \tau}\right|_{\tau=\tau_{j}} \neq 0
$$

provided that $d h\left(\omega_{0}^{2}\right) / d z \neq 0$, where $h(z)$ is defined by (2.8).

For the above $\varepsilon>0$, we define the set

$$
\Omega_{\varepsilon, 2 \pi / \omega_{0}}=\left\{(v, p): 0<v<\varepsilon,\left|p-\frac{2 \pi}{\omega_{0}}\right|<\varepsilon\right\} .
$$

It is easily to verify on $\left[\tau_{j}-\delta, \tau_{j}+\delta\right] \times \partial \Omega_{\varepsilon, 2 \pi / \omega_{0}}$ that the following condition holds.

(A4) det $\triangle(0, \tau, p)(v+(2 \pi / p) i)=0$ if and only if $v=0, \tau=\tau_{j}$, and $p=2 \pi / \omega_{0}, j=$ $0,1,2, \ldots$

Furthermore, the conditions (A5) and (A6) in [11] are obvious. 
In the following, we define

$$
H^{ \pm}\left(0, \tau_{j}, \frac{2 \pi}{\omega_{0}}\right)(v, p)=\operatorname{det} \triangle\left(0, \tau_{j} \pm \delta, \frac{2 \pi}{\omega_{0}}\right)\left(v+i \frac{2 \pi}{p}\right)
$$

then from (A4) we know that $H^{ \pm}\left(0, \tau_{j}, 2 \pi / \omega_{0}\right) \neq 0$ on $\partial \Omega_{\varepsilon, 2 \pi / \omega_{0}}$. Thus, the first crossing number $\gamma\left(0, \tau_{j}, 2 \pi / \omega_{0}\right)$ of the isolated center $\left(0, \tau_{j}, 2 \pi / \omega_{0}\right)$ can be defined as in the following form:

$$
\begin{aligned}
\gamma\left(0, \tau_{j}, \frac{2 \pi}{\omega_{0}}\right)= & \operatorname{deg}_{B}\left(H^{-}\left(0, \tau_{j}, \frac{2 \pi}{\omega_{0}}\right), 0, \Omega_{\varepsilon, 2 \pi / \omega_{0}}\right) \\
& -\operatorname{deg}_{B}\left(H^{+}\left(0, \tau_{j}, \frac{2 \pi}{\omega_{0}}\right), 0, \Omega_{\varepsilon, 2 \pi / \omega_{0}}\right),
\end{aligned}
$$

then $\gamma\left(0, \tau_{j}, 2 \pi / \omega_{0}\right)=1(-1)$ provided that $d h\left(\omega_{0}^{2}\right) / d z>0(<0)$, where $h(z)$ is defined by (2.8).

In what follows, we define

$$
\begin{gathered}
\Sigma=\operatorname{Cl}\left\{(z, \tau, p) \in X \times \mathbb{R}_{+} \times \mathbb{R}_{+}: z \text { is a } p \text {-periodic solution of }(3.2)\right\}, \\
N=\{(\bar{z}, \bar{\tau}, \bar{p}): F(\bar{z}, \bar{\tau}, \bar{p})=0\},
\end{gathered}
$$

and let $C\left(0, \tau_{j}, 2 \pi / \omega_{0}\right)$ denote the connected component of $\left(0, \tau_{j}, 2 \pi / \omega_{0}\right)$ in $\Sigma$.

From the above discussion, we have

$$
\sum_{(\bar{z}, \bar{\tau}, \bar{p}) \in C\left(0, \tau_{j}, 2 \pi / \omega_{0}\right) \cap N} \gamma(\bar{z}, \bar{\tau}, \bar{p}) \neq 0
$$

if $d h\left(\omega_{0}^{2}\right) / d z \neq 0$. Thus the connected component $C\left(0, \tau_{j}, 2 \pi / \omega_{0}\right)$ through $\left(0, \tau_{j}, 2 \pi / \omega_{0}\right)$ in $\Sigma$ is nonempty. Since the first crossing number of the unique equilibrium $(0,0,0,0)$ is not equal to zero, by [11, Theorem 3.3], we conclude that $C\left(0, \tau_{j}, 2 \pi / \omega_{0}\right)$ is unbounded. We thus have proved the following lemma.

Lemma 3.1. $C\left(0, \tau_{j}, 2 \pi / \omega_{0}\right)$ is unbounded for each center $\left(0, \tau_{j}, 2 \pi / \omega_{0}\right)$ if $d h\left(\omega_{0}^{2}\right) / d z \neq 0$.

Lemma 3.2. Assume that the condition $\left(\mathrm{H}_{2}\right)$ holds. Then all solutions of system (2.1) are uniformly bounded.

Proof. Let $\ell=\sum_{j=2}^{4} L\left(\left|a_{1 j}\right|+\left|a_{j 1}\right|\right)$. Then all solutions of system (2.1) satisfy the differential inequalities of the form

$$
-\mu_{k} x_{k}(t)-\ell \leq \dot{x}_{k}(t) \leq-\mu_{k} x_{k}(t)+\ell, \quad k=1,2,3,4
$$

From these inequalities, one can easily obtain

$$
-\frac{\ell}{\mu_{k}}+\left(x_{k}(0)+\frac{\ell}{\mu_{k}}\right) e^{-\mu_{k} t} \leq x_{k}(t) \leq \frac{\ell}{\mu_{k}}+\left(x_{k}(0)-\frac{\ell}{\mu_{k}}\right) e^{-\mu_{k} t} .
$$


Noticing that $\mu_{k}>0$, it follows that

$$
-\frac{\ell}{\mu_{k}}-\left|x_{k}(0)+\frac{\ell}{\mu_{k}}\right| \leq x_{k}(t) \leq \frac{\ell}{\mu_{k}}+\left|x_{k}(0)-\frac{\ell}{\mu_{k}}\right| \quad \forall t \geq 0 .
$$

Thus we know that all solutions of system (2.1) are uniformly bounded. This completes the proof of Lemma 3.2.

In the sequel, by applying a high-dimensional Bendixson's criterion due to Li and Muldowney [6], we obtain sufficient conditions ensuring that system (2.1) with $\tau=0$ has no nonconstant periodic solutions.

We first recall the definition of a $k$ th exterior power or multiplicative compound of a matrix.

Definition 3.3. Let $A$ be an $n \times m$ matrix of real or complex numbers. Let $a_{r_{1}, \ldots, r_{k}, l_{1}, \ldots, l_{k}}$ be the minor of $A$ determined by the rows $\left(r_{1}, \ldots, r_{k}\right)$ and the columns $\left(l_{1}, \ldots, l_{k}\right), 1 \leq r_{1}<$ $r_{2} \ldots<r_{k} \leq n, 1 \leq l_{1}<l_{2} \ldots<l_{k} \leq m$. The $k$ th multiplicative compound matrix $A^{(k)}$ of $A$ is the $\left(\begin{array}{l}n \\ k\end{array}\right) \times\left(\begin{array}{l}m \\ k\end{array}\right)$ matrix whose entries, written in lexicographic order, are $a_{r_{1}, \ldots, r_{k}, l_{1}, \ldots, l_{k}}$.

When $n=m$, the additive compound matrices are defined in the following way.

Definition 3.4. Let $A$ be an $n \times n$ matrix. The $k$ th additive compound $A^{[k]}$ of $A$ is the $\left(\begin{array}{l}n \\ k\end{array}\right) \times\left(\begin{array}{l}n \\ k\end{array}\right)$ matrix given by

$$
A^{[k]}=\left.D(I+h A)^{(k)}\right|_{h=0},
$$

where $D$ denotes the derivative with respect to $h$.

If $B=A^{[k]}$, the following formula for $b_{r, l}$ can be deduced from (3.17). For any integer $r=1, \ldots,\left(\begin{array}{l}n \\ k\end{array}\right)$, let $(r)=\left(r_{1}, r_{2}, \ldots, r_{k}\right)$ be the $r$ th member in the lexicographic ordering of all $k$-tuples of integers such that $1 \leq r_{1}<r_{2}<\cdots<r_{k} \leq n$. Then

$$
b_{r, l}= \begin{cases}a_{r_{1}, r_{1}}+\cdots+a_{r_{k}, r_{k}} & \text { if }(r)=(l), \\ (-1)^{s+t} a_{r_{s}, l_{t}} & \text { if exactly one entry } r_{s} \text { in }(r) \text { does not appear in }(l) \\ & \text { and } l_{t} \text { does not appear in }(r), \\ 0 & \text { if }(r) \text { differs from }(l) \text { in two or more entries. }\end{cases}
$$

In the extreme cases when $k=1$ and $k=n$, we have $A^{[1]}=A$ and $A^{[n]}=\operatorname{tr}(A)$. When $n=4$, for the 2 nd additive compound matrix $A^{[2]}$ of $A$, we have

$$
A^{[2]}=\left[\begin{array}{cccccc}
a_{11}+a_{22} & a_{23} & a_{24} & -a_{13} & -a_{14} & 0 \\
a_{32} & a_{11}+a_{33} & a_{34} & a_{12} & 0 & -a_{14} \\
a_{42} & a_{43} & a_{11}+a_{44} & 0 & a_{12} & a_{13} \\
-a_{31} & a_{21} & 0 & a_{22}+a_{33} & a_{34} & -a_{24} \\
-a_{41} & 0 & a_{21} & a_{43} & a_{22}+a_{44} & a_{23} \\
0 & -a_{41} & a_{31} & -a_{42} & a_{32} & a_{33}+a_{44}
\end{array}\right] .
$$


A BAM neural network with delays

For any finite $n$, the system of ordinary differential equations

$$
\dot{u}=g(u), \quad u \in \mathbb{R}^{n}, g \in C^{1},
$$

whose second compound equation with respect to a solution $u\left(t, x_{0}\right) \in \Omega$, where $\Omega \subseteq \mathbb{R}^{n}$ is an open set, is defined as

$$
\dot{z}(t)=\left(\frac{\partial g}{\partial u}\left(u\left(t, x_{0}\right)\right)\right)^{[2]} z(t)
$$

where $\left((\partial g / \partial u)\left(u\left(t, x_{0}\right)\right)\right)^{[2]}$ is the second additive compound matrix of the Jacobian matrix of vector function $g(u)$ at $u\left(t, x_{0}\right)$.

Lemma 3.5 [6]. Let $\Omega \subseteq \mathbb{R}^{n}$ be a simply connected region. Assume that the family of linear systems

$$
z^{\prime}(t)=\left(\frac{\partial g}{\partial u}\left(u\left(t, x_{0}\right)\right)\right)^{[2]} z(t), \quad x \in \Omega
$$

is qui-uniformly asymptotically stable. Then

(i) $\Omega$ contains no simple closed invariant curves including periodic orbits, homoclinic, heterclinic cycles,

(ii) each semi-orbit in $\Omega$ converges to a single equilibrium.

In particular, if $\Omega$ is positively invariant and contains a unique equilibrium $\bar{x}$, then $\bar{x}$ is globally asymptotically stable in $\Omega$.

We now consider system (2.1) with $\tau=0$, that is,

$$
\begin{gathered}
\dot{u}_{1}(t)=-\mu_{1} u_{1}(t)+c_{21} f_{1}\left(u_{2}(t)\right)+c_{31} f_{1}\left(u_{3}(t)\right)+c_{41} f_{1}\left(u_{4}(t)\right), \\
\dot{u}_{2}(t)=-\mu_{2} u_{2}(t)+c_{12} f_{2}\left(u_{1}(t)\right), \\
\dot{u}_{3}(t)=-\mu_{3} u_{3}(t)+c_{13} f_{3}\left(u_{1}(t)\right), \\
\dot{u}_{4}(t)=-\mu_{4} u_{4}(t)+c_{14} f_{4}\left(u_{1}(t)\right) .
\end{gathered}
$$

Denote

$$
g\left(u_{1}, u_{2}, u_{3}, u_{4}\right)=\left(\begin{array}{c}
-\mu_{1} u_{1}+c_{21} f_{1}\left(u_{2}\right)+c_{31} f_{1}\left(u_{3}\right)+c_{41} f_{1}\left(u_{4}\right) \\
-\mu_{2} u_{2}+c_{12} f_{2}\left(u_{1}\right) \\
-\mu_{3} u_{3}+c_{13} f_{3}\left(u_{1}\right) \\
-\mu_{4} u_{4}+c_{14} f_{4}\left(u_{1}\right)
\end{array}\right)
$$


Then, we have

$$
\frac{\partial g}{\partial u}=\left[\begin{array}{cccc}
-\mu_{1} & c_{21} f_{1}^{\prime}\left(u_{2}\right) & c_{31} f_{1}^{\prime}\left(u_{3}\right) & c_{41} f_{1}^{\prime}\left(u_{4}\right) \\
c_{12} f_{2}^{\prime}\left(u_{1}\right) & -\mu_{2} & 0 & 0 \\
c_{13} f_{3}^{\prime}\left(u_{1}\right) & 0 & -\mu_{3} & 0 \\
c_{14} f_{4}^{\prime}\left(u_{1}\right) & 0 & 0 & -\mu_{4}
\end{array}\right]
$$

and, in view of (3.19),

$$
\begin{aligned}
& \left(\frac{\partial g}{\partial u}\right)^{[2]} \\
& =\left[\begin{array}{cccccc}
-\left(\mu_{1}+\mu_{2}\right) & 0 & 0 & -c_{31} f_{1}^{\prime}\left(u_{3}\right) & -c_{41} f_{1}^{\prime}\left(u_{4}\right) & 0 \\
0 & -\left(\mu_{1}+\mu_{3}\right) & 0 & c_{21} f_{1}^{\prime}\left(u_{2}\right) & 0 & -c_{41} f_{1}^{\prime}\left(u_{4}\right) \\
0 & 0 & -\left(\mu_{1}+\mu_{4}\right) & 0 & c_{21} f_{1}^{\prime}\left(u_{2}\right) & c_{31} f_{1}^{\prime}\left(u_{3}\right) \\
-c_{13} f_{3}^{\prime}\left(u_{1}\right) & c_{12} f_{2}^{\prime}\left(u_{1}\right) & 0 & -\left(\mu_{2}+\mu_{3}\right) & 0 & 0 \\
-c_{14} f_{4}^{\prime}\left(u_{1}\right) & 0 & c_{12} f_{2}^{\prime}\left(u_{1}\right) & 0 & -\left(\mu_{2}+\mu_{4}\right) & 0 \\
0 & -c_{14} f_{4}^{\prime}\left(u_{1}\right) & c_{13} f_{3}^{\prime}\left(u_{1}\right) & 0 & 0 & -\left(\mu_{3}+\mu_{4}\right)
\end{array}\right] .
\end{aligned}
$$

Therefore, the second compound system

$$
\left(\dot{z}_{1}, \dot{z}_{2}, \dot{z}_{3}, \dot{z}_{4}, \dot{z}_{5}, \dot{z}_{6}\right)^{\mathrm{T}}=\left(\frac{\partial g}{\partial u}\right)^{[2]}\left(z_{1}, z_{2}, z_{3}, z_{4}, z_{5}, z_{6}\right)^{\mathrm{T}}
$$

is

$$
\begin{aligned}
& \dot{z}_{1}=-\left(\mu_{1}+\mu_{2}\right) z_{1}-c_{31} f_{1}^{\prime}\left(u_{3}(t)\right) z_{4}-c_{41} f_{1}^{\prime}\left(u_{4}(t)\right) z_{5}, \\
& \dot{z}_{2}=-\left(\mu_{1}+\mu_{3}\right) z_{2}+c_{21} f_{1}^{\prime}\left(u_{2}(t)\right) z_{4}-c_{41} f_{1}^{\prime}\left(u_{4}(t)\right) z_{6}, \\
& \dot{z}_{3}=-\left(\mu_{1}+\mu_{4}\right) z_{3}+c_{21} f_{1}^{\prime}\left(u_{2}(t)\right) z_{5}+c_{31} f_{1}^{\prime}\left(u_{3}(t)\right) z_{6}, \\
& \dot{z}_{4}=-\left(\mu_{2}+\mu_{3}\right) z_{4}-c_{13} f_{3}^{\prime}\left(u_{1}(t)\right) z_{1}+c_{12} f_{2}^{\prime}\left(u_{1}(t)\right) z_{2}, \\
& \dot{z}_{5}=-\left(\mu_{2}+\mu_{4}\right) z_{5}-c_{14} f_{4}^{\prime}\left(u_{1}(t)\right) z_{1}+c_{12} f_{2}^{\prime}\left(u_{1}(t)\right) z_{3}, \\
& \dot{z}_{6}=-\left(\mu_{3}+\mu_{4}\right) z_{6}-c_{14} f_{4}^{\prime}\left(u_{1}(t)\right) z_{2}+c_{13} f_{3}^{\prime}\left(u_{1}(t)\right) z_{3},
\end{aligned}
$$

where $u(t)=\left(u_{1}(t), u_{2}(t), u_{3}(t), u_{4}(t)\right)^{\mathrm{T}}$ is a solution of the system $(3.20)$ with $u(0)=u_{0} \in$ $\mathbb{R}^{4}$. Set

$$
W(z)=\max \left\{\kappa_{l}\left|z_{l}\right|: l=1,2, \ldots, 6\right\}
$$


where $\kappa_{l}>0(l=1,2, \ldots, 6)$ are constants. Then direct calculation leads to the following inequalities:

$$
\begin{aligned}
& \frac{d^{+}}{d t} \kappa_{1}\left|z_{1}\right| \leq-\left(\mu_{1}+\mu_{2}\right) \kappa_{1}\left|z_{1}\right|+\frac{\kappa_{1}}{\kappa_{4}}\left|c_{31} f_{1}^{\prime}\left(u_{3}(t)\right)\right| \kappa_{4}\left|z_{4}\right|+\frac{\kappa_{1}}{\kappa_{5}}\left|c_{41} f_{1}^{\prime}\left(u_{4}(t)\right)\right| \kappa_{5}\left|z_{5}\right|, \\
& \frac{d^{+}}{d t} \kappa_{2}\left|z_{2}\right| \leq-\left(\mu_{1}+\mu_{3}\right) \kappa_{2}\left|z_{2}\right|+\frac{\kappa_{2}}{\kappa_{4}}\left|c_{21} f_{1}^{\prime}\left(u_{2}(t)\right)\right| \kappa_{4}\left|z_{4}\right|+\frac{\kappa_{2}}{\kappa_{6}}\left|c_{41} f_{1}^{\prime}\left(u_{4}(t)\right)\right| \kappa_{6}\left|z_{6}\right|, \\
& \frac{d^{+}}{d t} \kappa_{3}\left|z_{3}\right| \leq-\left(\mu_{1}+\mu_{4}\right) \kappa_{3}\left|z_{3}\right|+\frac{\kappa_{3}}{\kappa_{5}}\left|c_{21} f_{1}^{\prime}\left(u_{2}(t)\right)\right| \kappa_{5}\left|z_{5}\right|+\frac{\kappa_{3}}{\kappa_{6}}\left|c_{31} f_{1}^{\prime}\left(u_{3}(t)\right)\right| \kappa_{6}\left|z_{6}\right|, \\
& \frac{d^{+}}{d t} \kappa_{4}\left|z_{4}\right| \leq-\left(\mu_{2}+\mu_{3}\right) \kappa_{4}\left|z_{4}\right|+\frac{\kappa_{4}}{\kappa_{1}}\left|c_{13} f_{3}^{\prime}\left(u_{1}(t)\right)\right| \kappa_{1}\left|z_{1}\right|+\frac{\kappa_{4}}{\kappa_{2}}\left|c_{12} f_{2}^{\prime}\left(u_{1}(t)\right)\right| \kappa_{2}\left|z_{2}\right|, \\
& \frac{d^{+}}{d t} \kappa_{5}\left|z_{5}\right| \leq-\left(\mu_{2}+\mu_{4}\right) \kappa_{5}\left|z_{5}\right|+\frac{\kappa_{5}}{\kappa_{1}}\left|c_{14} f_{4}^{\prime}\left(u_{1}(t)\right)\right| \kappa_{1}\left|z_{1}\right|+\frac{\kappa_{5}}{\kappa_{3}}\left|c_{12} f_{2}^{\prime}\left(u_{1}(t)\right)\right| \kappa_{3}\left|z_{3}\right|, \\
& \frac{d^{+}}{d t} \kappa_{6}\left|z_{6}\right| \leq-\left(\mu_{3}+\mu_{4}\right) \kappa_{6}\left|z_{6}\right|+\frac{\kappa_{6}}{\kappa_{2}}\left|c_{14} f_{4}^{\prime}\left(u_{1}(t)\right)\right| \kappa_{2}\left|z_{2}\right|+\frac{\kappa_{6}}{\kappa_{3}}\left|c_{13} f_{3}^{\prime}\left(u_{1}(t)\right)\right| \kappa_{3}\left|z_{3}\right|,
\end{aligned}
$$

where $d^{+} / d t$ denotes the upper right-hand derivative. Consequently, we have

$$
\frac{d^{+}}{d t} W(z(t)) \leq \rho(t) W(z(t))
$$

with

$$
\begin{aligned}
\rho(t)=\max \{ & -\left(\mu_{1}+\mu_{2}\right)+\frac{\kappa_{1}}{\kappa_{4}}\left|c_{31} f_{1}^{\prime}\left(u_{3}(t)\right)\right|+\frac{\kappa_{1}}{\kappa_{5}}\left|c_{41} f_{1}^{\prime}\left(u_{4}(t)\right)\right|, \\
& -\left(\mu_{1}+\mu_{3}\right)+\frac{\kappa_{2}}{\kappa_{4}}\left|c_{21} f_{1}^{\prime}\left(u_{2}(t)\right)\right|+\frac{\kappa_{2}}{\kappa_{6}}\left|c_{41} f_{1}^{\prime}\left(u_{4}(t)\right)\right|, \\
& -\left(\mu_{1}+\mu_{4}\right)+\frac{\kappa_{3}}{\kappa_{5}}\left|c_{21} f_{1}^{\prime}\left(u_{2}(t)\right)\right|+\frac{\kappa_{3}}{\kappa_{6}}\left|c_{31} f_{1}^{\prime}\left(u_{3}(t)\right)\right|, \\
& -\left(\mu_{2}+\mu_{3}\right)+\frac{\kappa_{4}}{\kappa_{1}}\left|c_{13} f_{3}^{\prime}\left(u_{1}(t)\right)\right|+\frac{\kappa_{4}}{\kappa_{2}}\left|c_{12} f_{2}^{\prime}\left(u_{1}(t)\right)\right|, \\
& -\left(\mu_{2}+\mu_{4}\right)+\frac{\kappa_{5}}{\kappa_{1}}\left|c_{14} f_{4}^{\prime}\left(u_{1}(t)\right)\right|+\frac{\kappa_{5}}{\kappa_{3}}\left|c_{12} f_{2}^{\prime}\left(u_{1}(t)\right)\right|, \\
& \left.-\left(\mu_{3}+\mu_{4}\right)+\frac{\kappa_{6}}{\kappa_{2}}\left|c_{14} f_{4}^{\prime}\left(u_{1}(t)\right)\right|+\frac{\kappa_{6}}{\kappa_{3}}\left|c_{13} f_{3}^{\prime}\left(u_{1}(t)\right)\right|\right\} .
\end{aligned}
$$

For the sake of convenience of discussion, we further make the following hypothesis. 
$\left(\mathrm{H}_{5}\right)$ There exist constants $\kappa_{l}>0(l=1,2, \ldots, 6)$ such that

$$
\begin{aligned}
\sup _{u \in \mathbb{R}}\{ & -\left(\mu_{1}+\mu_{2}\right)+\frac{\kappa_{1}}{\kappa_{4}}\left|c_{31} f_{1}^{\prime}(u)\right|+\frac{\kappa_{1}}{\kappa_{5}}\left|c_{41} f_{1}^{\prime}(u)\right|, \\
& -\left(\mu_{1}+\mu_{3}\right)+\frac{\kappa_{2}}{\kappa_{4}}\left|c_{21} f_{1}^{\prime}(u)\right|+\frac{\kappa_{2}}{\kappa_{6}}\left|c_{41} f_{1}^{\prime}(u)\right|, \\
& -\left(\mu_{1}+\mu_{4}\right)+\frac{\kappa_{3}}{\kappa_{5}}\left|c_{21} f_{1}^{\prime}(u)\right|+\frac{\kappa_{3}}{\kappa_{6}}\left|c_{31} f_{1}^{\prime}(u)\right|, \\
& -\left(\mu_{2}+\mu_{3}\right)+\frac{\kappa_{4}}{\kappa_{1}}\left|c_{13} f_{3}^{\prime}(u)\right|+\frac{\kappa_{4}}{\kappa_{2}}\left|c_{12} f_{2}^{\prime}(u)\right|, \\
& -\left(\mu_{2}+\mu_{4}\right)+\frac{\kappa_{5}}{\kappa_{1}}\left|c_{14} f_{4}^{\prime}(u)\right|+\frac{\kappa_{5}}{\kappa_{3}}\left|c_{12} f_{2}^{\prime}(u)\right|, \\
& \left.-\left(\mu_{3}+\mu_{4}\right)+\frac{\kappa_{6}}{\kappa_{2}}\left|c_{14} f_{4}^{\prime}(u)\right|+\frac{\kappa_{6}}{\kappa_{3}}\left|c_{13} f_{3}^{\prime}(u)\right|\right\}<0 .
\end{aligned}
$$

Thus, we have the following result.

Lemma 3.6. If the hypotheses $\left(\mathrm{H}_{1}\right),\left(\mathrm{H}_{2}\right)$, and $\left(\mathrm{H}_{5}\right)$ hold, then the system (3.23) has no nonconstant periodic solution. Furthermore, the unique equilibrium $(0,0,0,0)$ is globally asymptotically stable in $\mathbb{R}^{4}$.

Indeed, in this case, by the boundedness of solution to (3.23), there exists $\delta>0$ such that $\rho(t) \leq-\delta<0$, and thus

$$
W(z(t)) \leq W(z(s)) e^{-\delta(t-s)}, \quad t \geq s \geq 0 .
$$

This shows that the second compound system (3.28) is qui-uniform asymptotically stable, and from Lemma 3.5, the results of Lemma 3.6 can be obtained.

Lemma 3.7. If the conditions $\left(\mathrm{H}_{1}\right),\left(\mathrm{H}_{2}\right)$, and $\left(\mathrm{H}_{5}\right)$ hold, then the periods of the periodic solution of the (2.1) are uniformly bounded.

Proof. Suppose $u(t)=\left(u_{1}(t), u_{2}(t), u_{3}(t), u_{4}(t)\right)^{\mathrm{T}}$ is a $\tau$-periodic solution of system (2.1), then $u(t)$ is a $\tau$-periodic solution of the ordinary differential equation system (3.23) with a unique equilibrium $u=0$. By Lemma 3.6, system (3.23) has no nonconstant periodic solutions. Therefore, system (2.1) has no nonconstant $\tau$-periodic solution and thus the proof is complete.

In the following we state and prove our main result in this section.

Theorem 3.8. Suppose that the conditions $\left(\mathrm{H}_{1}\right)-\left(\mathrm{H}_{5}\right)$ hold. Then for each $\tau>\tau_{j}, j=1,2, \ldots$, system (2.1) has at least $j$ nonconstant periodic solutions.

Proof. It is sufficient to prove that the connected component $C\left(0, \tau_{j}, 2 \pi / \omega_{0}\right)$ onto $\tau$-space is $[\bar{\tau}, \infty)$ for each $j \geq 1$, where $\bar{\tau} \leq \tau_{j}$. 
A BAM neural network with delays

From the discussion in Section 2, we have

$$
\frac{2 \pi}{\omega_{0}}<\tau_{j} \text { for } j>0 .
$$

From Lemma 3.6, we know that system (2.1) with $\tau=0$ has no nontrivial periodic solution. Consequently, the projection of $C\left(0, \tau_{j}, 2 \pi / \omega_{0}\right)$ onto $\tau$-space is away from zero.

Suppose that the projection of $C\left(0, \tau_{j}, 2 \pi / \omega_{0}\right)$ onto $\tau$-space is bounded, that is, there exists $\tau^{*}>0$ such that the projection of $C\left(0, \tau_{j}, 2 \pi / \omega_{0}\right)$ onto $\tau$-space is contained in interval $\left(0, \tau^{*}\right)$. Since $2 \pi / \omega_{0}<\tau_{j}$ and applying Lemma 3.7, one can obtain $0<p<\tau^{*}$ for $(u(t), \tau, p) \in C\left(0, \tau_{j}, 2 \pi / \omega_{0}\right)$. Therefore, the projection of $C\left(0, \tau_{j}, 2 \pi / \omega_{0}\right)$ onto $p$-space is also bounded. Thus, we get together with Lemma 3.2 that the connected component $C\left(0, \tau_{j}, 2 \pi / \omega_{0}\right)$ is bounded. This contradicts with Lemma 3.1 and the proof is complete.

\section{An example and numerical simulations}

4.1. An example. Consider the four-neuron BAM neural network with two delays described by the following functional differential equations:

$$
\begin{aligned}
\dot{x}_{1}(t)=-\mu x_{1}(t)+c_{21} f\left(x_{2}\left(t-\tau_{2}\right)\right)+c_{31} f\left(x_{3}\left(t-\tau_{2}\right)\right)+c_{41} f\left(x_{4}\left(t-\tau_{2}\right)\right), \\
\dot{x}_{2}(t)=-\mu x_{2}(t)+c_{12} f\left(x_{1}\left(t-\tau_{1}\right)\right), \\
\dot{x}_{3}(t)=-\mu x_{3}(t)+c_{13} f\left(x_{1}\left(t-\tau_{1}\right)\right), \\
\dot{x}_{4}(t)=-\mu x_{4}(t)+c_{14} f\left(x_{1}\left(t-\tau_{1}\right)\right),
\end{aligned}
$$

with $\mu>0, c_{k 1}(k=2,3,4)>0$, and $c_{1 l}(l=2,3,4)<0$. In addition, the activation function $f$ satisfies the following condition.

$\left(\mathrm{H}_{6}\right) f \in C^{2}(\mathbb{R}, \mathbb{R}), f(0)=0$, and $(0,0,0,0)$ is the unique equilibrium of (4.1).

$\left(\mathrm{H}_{7}\right)$ There are positive constants $L>0$ such that $|f(x)| \leq L$ for all $x \in \mathbb{R}$, and

$$
\mu^{4}<\left(c_{12} c_{21}+c_{13} c_{31}+c_{14} c_{41}\right)^{2} f^{\prime 4}(0)<2 \mu^{4} .
$$

$\left(\mathrm{H}_{8}\right)\left(\left|c_{21}\right|+\left|c_{31}\right|\right)\left|f^{\prime}(x)\right|<2 \mu,\left(\left|c_{21}\right|+\left|c_{41}\right|\right)\left|f^{\prime}(x)\right|<2 \mu,\left(\left|c_{31}\right|+\left|c_{41}\right|\right)\left|f^{\prime}(x)\right|<2 \mu$, $\left(\left|c_{12}\right|+\left|c_{13}\right|\right)\left|f^{\prime}(x)\right|<2 \mu,\left(\left|c_{12}\right|+\left|c_{14}\right|\right)\left|f^{\prime}(x)\right|<2 \mu,\left(\left|c_{13}\right|+\left|c_{14}\right|\right)\left|f^{\prime}(x)\right|<2 \mu$, for $x \in \mathbb{R}$.

Under the assumption $\left(\mathrm{H}_{7}\right)$, the following equation:

$$
z^{4}+a z^{3}+b z^{2}+c z+d=0
$$

has only one positive $z_{0}$ since

$$
\begin{gathered}
a=4 \mu^{2}>0, \\
b=6 \mu^{4}-\left(c_{12} c_{21}+c_{13} c_{31}+c_{14} c_{41}\right)^{2} f^{\prime 4}(0)>0, \\
c=4 \mu^{6}-2 \mu^{2}\left(c_{12} c_{21}+c_{13} c_{31}+c_{14} c_{41}\right)^{2} f^{\prime 4}(0)>0, \\
d=\mu^{8}-\mu^{4}\left(c_{12} c_{21}+c_{13} c_{31}+c_{14} c_{41}\right)^{2} f^{\prime 4}(0)<0 .
\end{gathered}
$$


Let $\omega_{0}=\sqrt{z_{0}}$ and define

$$
\tau_{j}=\frac{1}{\omega_{0}}\left\{\arccos \left(\frac{\mu^{2}-\omega_{0}^{2}}{\left(c_{12} c_{21}+c_{13} c_{31}+c_{14} c_{41}\right) f^{\prime 2}(0)}\right)+2 j \pi\right\}, \quad j=0,1, \ldots
$$

Set $\tau=\tau_{1}+\tau_{2}$ and take $\kappa_{1}=\kappa_{2}=\cdots=\kappa_{6}>0$ in $\left(\mathrm{H}_{4}\right)$. From Theorem 3.8, we have the following result for system (4.1).

Theorem 4.1. Suppose that $\left(\mathrm{H}_{6}\right)-\left(\mathrm{H}_{8}\right)$ are satisfied. Then system (4.1) has at least $j$ nonconstant periodic solutions when $\tau>\tau_{j}, j \geq 1$.

In particular, when $\mu=1$ and $f(u)=\tanh (u)$ in system (4.1), we have the following corollary.

COROLlary 4.2. For the BAM neural network model with four neurons,

$$
\begin{gathered}
\dot{x}_{1}(t)=-x_{1}(t)+c_{21} \tanh \left(x_{2}\left(t-\tau_{2}\right)\right)+c_{31} \tanh \left(x_{3}\left(t-\tau_{2}\right)\right)+c_{41} \tanh \left(x_{4}\left(t-\tau_{2}\right)\right), \\
\dot{x}_{2}(t)=-x_{2}(t)+c_{12} \tanh \left(x_{1}\left(t-\tau_{1}\right)\right), \\
\dot{x}_{3}(t)=-x_{3}(t)+c_{13} \tanh \left(x_{1}\left(t-\tau_{1}\right)\right), \\
\dot{x}_{4}(t)=-x_{4}(t)+c_{14} \tanh \left(x_{1}\left(t-\tau_{1}\right)\right),
\end{gathered}
$$

if $\left|c_{21}\right|+\left|c_{31}\right|<2,\left|c_{21}\right|+\left|c_{41}\right|<2,\left|c_{31}\right|+\left|c_{41}\right|<2,\left|c_{12}\right|+\left|c_{13}\right|<2,\left|c_{12}\right|+\left|c_{14}\right|<2,\left|c_{13}\right|+$ $\left|c_{14}\right|<2$, and

$$
1<\left(c_{12} c_{21}+c_{13} c_{31}+c_{14} c_{41}\right)^{2}<2
$$

is satisfied. Then system (4.6) has at least $j$ nonconstant periodic solutions when $\tau>\tau_{j}$, $j \geq 1$, and $\tau_{j}$ is defined in (4.5) with $f^{\prime}(0)=1$.

4.2. Numerical simulations. In this subsection, we give numerical simulations supporting our theoretical analysis. As an example, we consider the following system:

$$
\begin{gathered}
\dot{x}_{1}(t)=-2 x_{1}(t)+2 \tanh \left(x_{2}\left(t-\tau_{2}\right)\right)+\tanh \left(x_{3}\left(t-\tau_{2}\right)\right)+\tanh \left(x_{4}\left(t-\tau_{2}\right)\right), \\
\dot{x}_{2}(t)=-2 x_{2}(t)-\tanh \left(x_{1}\left(t-\tau_{1}\right)\right), \\
\dot{x}_{3}(t)=-2 x_{3}(t)-2 \tanh \left(x_{1}\left(t-\tau_{1}\right)\right), \\
\dot{x}_{4}(t)=-2 x_{4}(t)-\tanh \left(x_{1}\left(t-\tau_{1}\right)\right),
\end{gathered}
$$



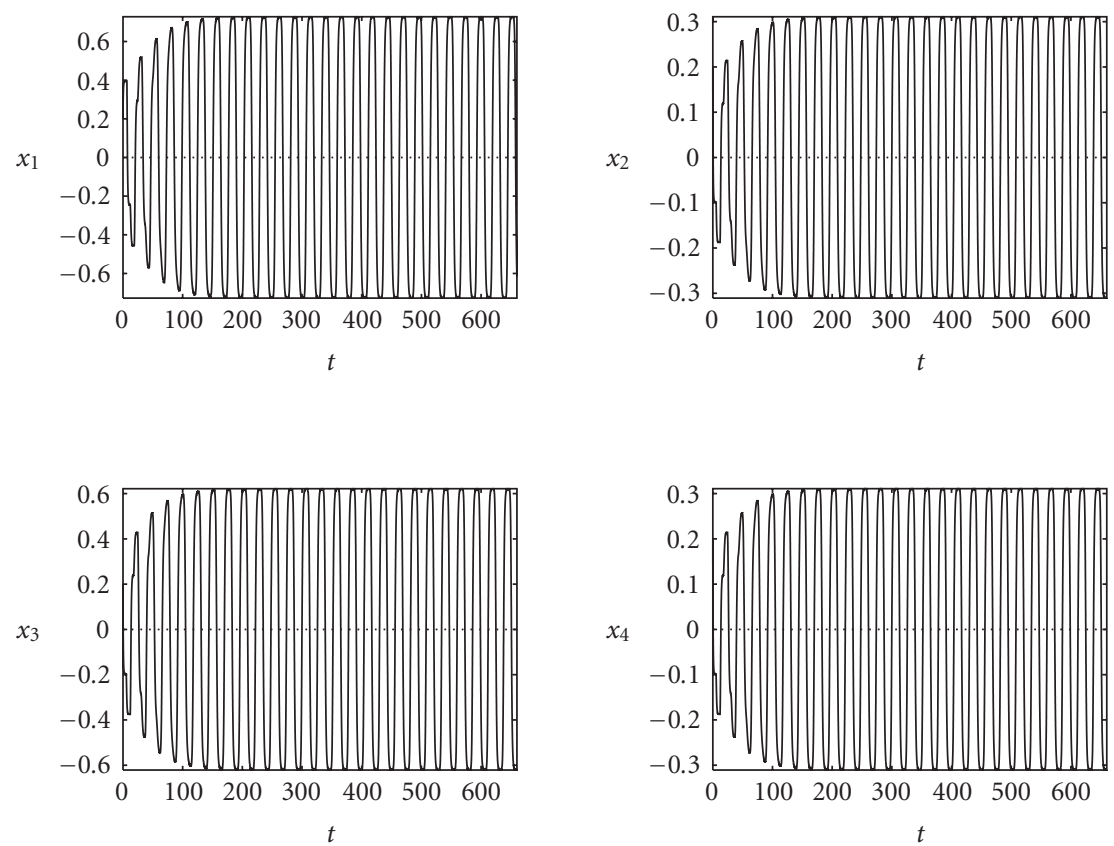

Figure 4.1. The trajectories graphs of system (4.8) with $\tau_{1}=5.45, \tau_{2}=6.55$, and initial data $x_{1}(t)=$ $x_{2}(t)=x_{3}(t)=x_{4}(t)=0.2, t \in[-6.55,0]$.

which has a unique equilibrium $(0,0,0,0)$. It follows from (4.4) that $a=16, b=71, c=$ $56, d=-144$ since $f^{\prime}(0)=\tanh ^{\prime}(0)=1$. Thus, in this case,

$$
h(z)=z^{4}+16 z^{3}+71 z^{2}+56 z-144
$$

it is easy to see that equation $h(z)=0$ has only a positive root $z_{0}=1$, and so $\omega_{0}=1$. From (4.5), we have

$$
\tau_{j}=\arccos \left(-\frac{3}{5}\right)+2 j \pi, \quad j=0,1, \ldots
$$

Clearly, conditions $\left(\mathrm{H}_{6}\right)-\left(\mathrm{H}_{8}\right)$ hold. Therefore, from Theorem 4.1, system (4.8) has at least $j$ nonconstant periodic solutions when $\tau>\tau_{j}, j \geq 1$. From (4.10), we can compute $\tau_{0}=2.2143, \tau_{1}=8.4975, \tau_{2}=14.7807, \tau_{3}=21.0639, \tau_{4}=27.3470, \tau_{4}=33.6302$. The simulations consistently show global existence of periodic solution: existence of large amplitude periodic solutions for values of $\tau=\tau_{1}+\tau_{2}$ far away from $\tau_{j}$. That the delays are chosen as $\tau_{1}=5.45, \tau=6.55$ such that $\tau=\tau_{1}+\tau_{2}$ is between the two Hopf bifurcation values $\tau_{1}=8.4975$ and $\tau_{2}=14.7807$ is shown in Figure 4.1 and the case that $\tau_{1}=15.46$ and $\tau_{2}=16.78$ is shown in Figure 4.2. 

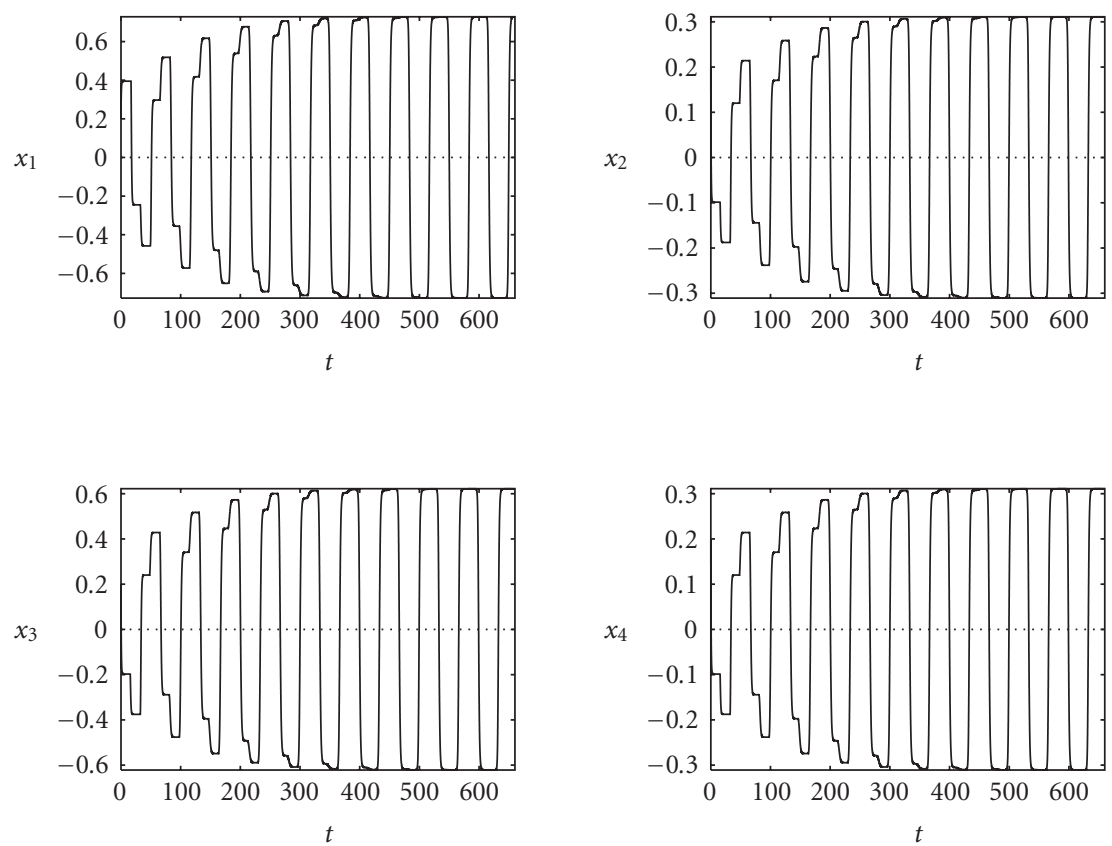

Figure 4.2. The trajectories graphs of system (4.8) with $\tau_{1}=15.46, \tau_{2}=16.78$, and initial data $x_{1}(t)=$ $x_{2}(t)=x_{3}(t)=x_{4}(t)=0.2, t \in[-16.78,0]$.

\section{Acknowledgment}

This research was supported by the NNSF of China (10571078) and the Teaching and Research Award Program for Outstanding Young Teachers in Higher Education Institutions of the Ministry of Education of China.

\section{References}

[1] S. A. Campbell, S. Ruan, and J. Wei, Qualitative analysis of a neural network model with multiple time delays, International Journal of Bifurcation and Chaos in Applied Sciences and Engineering 9 (1999), no. 8, 1585-1595.

[2] T. Faria, On a planar system modelling a neuron network with memory, Journal of Differential Equations 168 (2000), no. 1, 129-149.

[3] K. Gopalsamy and X.-Z. He, Delay-independent stability in bidirectional associative memory networks, IEEE Transactions on Neural Networks 5 (1994), no. 6, 998-1002.

[4] K. Gopalsamy and I. Leung, Delay induced periodicity in a neural netlet of excitation and inhibition, Physica D: Nonlinear Phenomena 89 (1996), no. 3-4, 395-426.

[5] B. D. Hassard, N. D. Kazarinoff, and Y. H. Wan, Theory and Applications of Hopf Bifurcation, London Mathematical Society Lecture Note Series, vol. 41, Cambridge University Press, Cambridge, 1981.

[6] Y. Li and J. S. Muldowney, On Bendixson's criterion, Journal of Differential Equations 106 (1993), no. $1,27-39$. 
18 A BAM neural network with delays

[7] S. Ruan and J. Wei, On the zeros of a third degree exponential polynomial with applications to a delayed model for the control of testosterone secretion, IMA Journal of Mathematics Applied in Medicine and Biology 18 (2001), no. 1, 41-52.

[8] J. Wei and M. Y. Li, Global existence of periodic solutions in a tri-neuron network model with delays, Physica D: Nonlinear Phenomena 198 (2004), no. 1-2, 106-119.

[9] J. Wei and S. Ruan, Stability and bifurcation in a neural network model with two delays, Physica D: Nonlinear Phenomena 130 (1999), no. 3-4, 255-272.

[10] J. Wei and M. G. Velarde, Bifurcation analysis and existence of periodic solutions in a simple neural network with delays, Chaos 14 (2004), no. 3, 940-953.

[11] J. Wu, Symmetric functional-differential equations and neural networks with memory, Transactions of the American Mathematical Society 350 (1998), no. 12, 4799-4838.

[12] X.-P. Yan and W.-T. Li, Stability and bifurcation in a simplified four-neuron BAM neural network with multiple delays, Discrete Dynamics in Nature and Society 2006 (2006), Article ID 32529, $1-29$.

Xiang-Ping Yan: School of Mathematics and Statistics, Lanzhou University, Lanzhou 730000, China; School of Mathematics, Physics, and Software Engineering, Lanzhou Jiaotong University, Lanzhou 730070, China

E-mail address: yanxp@mail.lzjtu.cn

Wan-Tong Li: School of Mathematics and Statistics, Lanzhou University, Lanzhou 730000, China E-mail address: wtli@lzu.edu.cn 


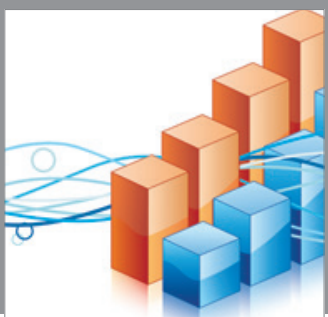

Advances in

Operations Research

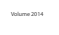



\section{The Scientific} World Journal
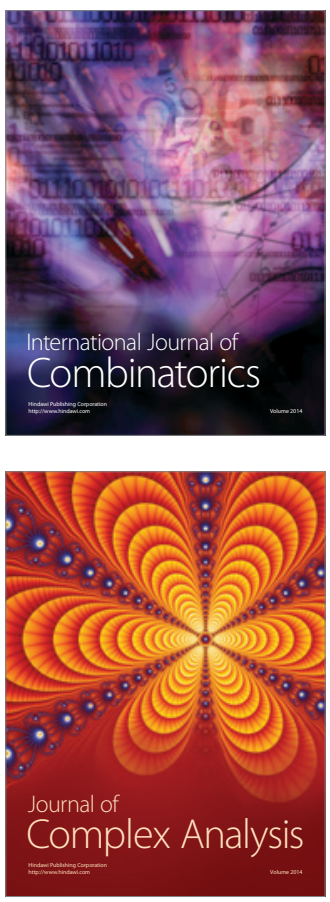

International Journal of

Mathematics and

Mathematical

Sciences
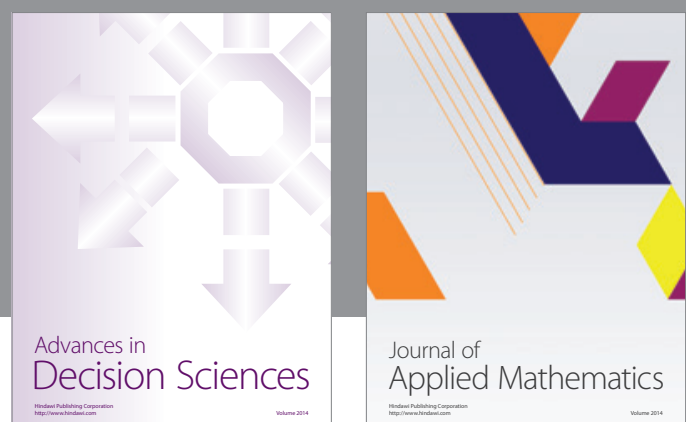

Journal of

Applied Mathematics
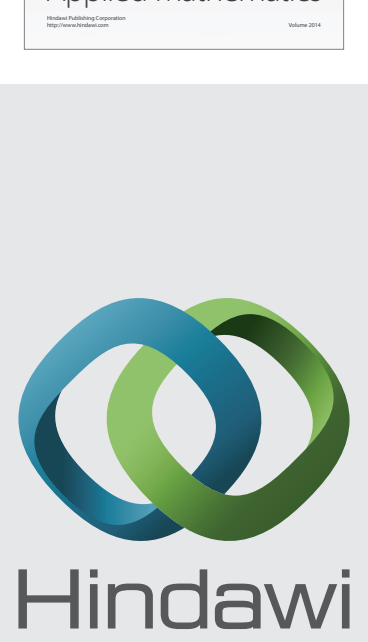

Submit your manuscripts at http://www.hindawi.com
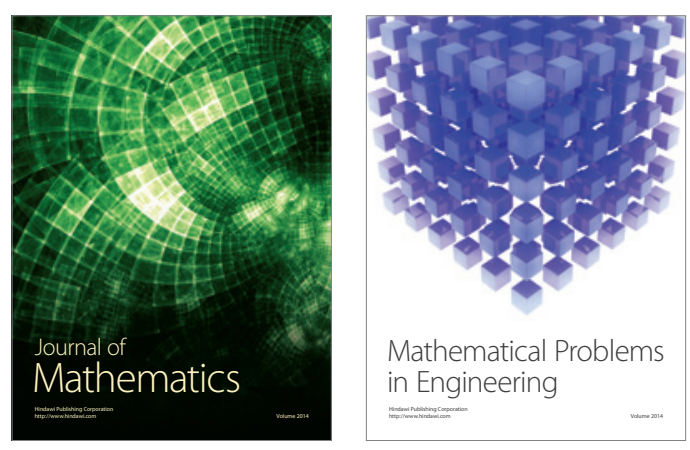

Mathematical Problems in Engineering
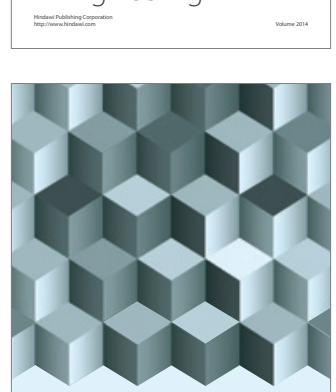

Journal of

Function Spaces
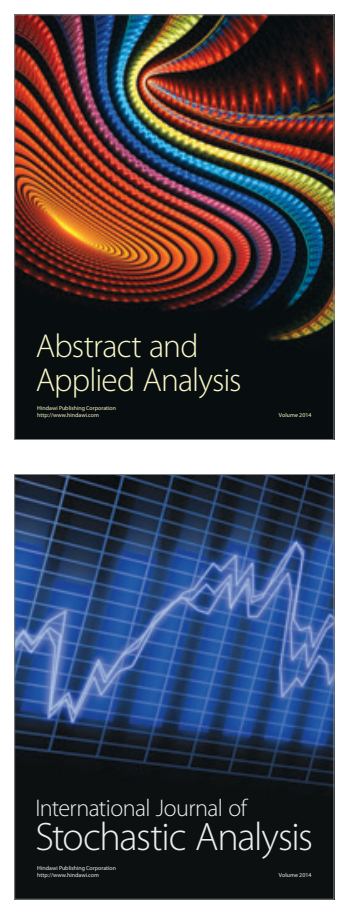

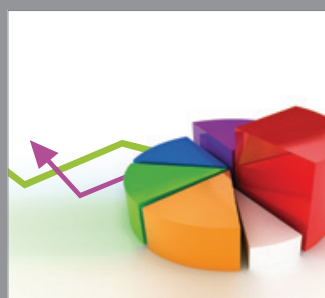

ournal of

Probability and Statistics

Promensencen
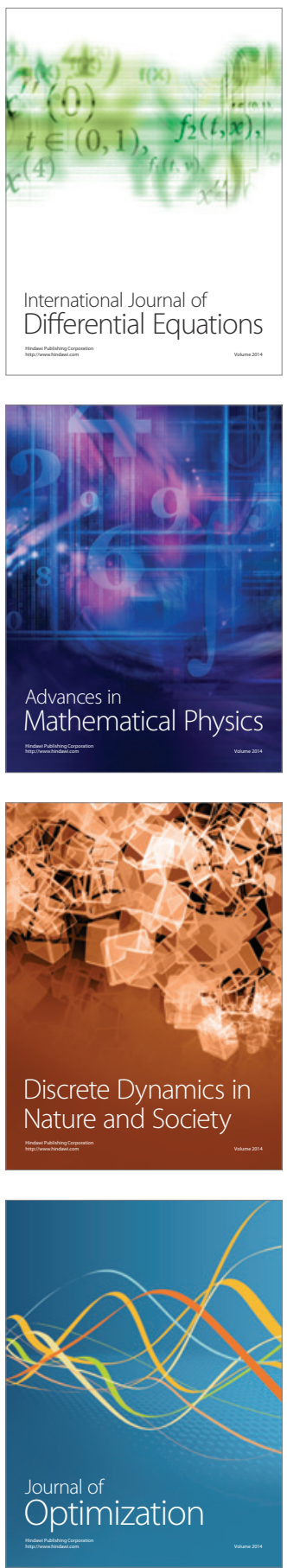